\title{
FRANÇOIS BON ET LA «DICTION DU MONDE»
}

\begin{abstract}
Roche Anne, François Bon et la «diction du monde» [François Bon, "phirasing the world"]. Studia Romanica Posnaniensia, Adam Mickiewicz University Press, Poznań, vol. XXXI: 2004, pp. 83-96. ISBN 83-232-1353-4, ISSN 0137-2475.

François Bon's novels seem to deal with marginal characters and subjects: unemployment, wreckage of industry, drugs, petty crimes, prison... His work is actually a lyrical eulogy to a disappearing modernity, but reveals a sophisticated formal research: it shapes itself on the I mould of Greek tragedy, while kneading more current forms of language.
\end{abstract}

François Bon n'est certes pas un auteur inconnu du public français, mais son œuvre est peut-être l'objet d'un malentendu, comme l'indiquent certains articles qui lui sont consacrés dans les pages littéraires des grands quotidiens. Ainsi Pierre Lepape, du «Monde», intitule Dépôts de mémoire l'article qu'il consacre à C'était toute une vie ${ }^{1}$ : d'après lui, nous serions en train de vivre la fin de l'histoire - au sens hegelien - ce qui s'accompagnerait de la fin d'un certain type de roman, «et sans avenir pensable, à quoi bon s'encombrer du passé, si ce n'est sous les formes malingres de l'autobiographie et du mémorial? $»^{2}$ Le joumaliste suggère donc que le roman de François Bon est autobiographique - ce qui peut se concevoir, mais pas forcément sous cette forme quelque peu dédaigneuse, - et qu'il n'est que tourné vers la commémoration d'un monde passé, ce qu'il faudra également examiner. Mémorial s'entend aussi par rapport à la jeune femme morte qui est le principal personnage du livre, marginale, droguée, et morte d'une overdose. A propos du même roman, la journaliste Michèle Gazier intitule Les porte-paroles la chronique qu'elle consacre à François Bon et au roman de Lydie Salvayre, La Puissance des mouches, histoire d'un gardien de musée condamné pour meurtre ${ }^{3}$ : ici encore, le roman de François Bon, ainsi mis en contexte, est présenté comme consacré à des personnages hors société, et l'analyse ne souligne que son contenu. Citons enfin

\footnotetext{
'F. B on, C'était toute une vie, Editions Verdier, 1995.

${ }^{2}$ P. Lepape, Dépôts de mémoire, «Le Monde» du 6 octobre 1995.

${ }^{3}$ M. Gazier, Les porte-paroles, «Télérama» du 27 septembre 1995.
} 
Alain André, animateur d'ateliers d'écriture et écrivain, qui écrit à propos de Sang gris $^{4}$ : «François Bon est aussi de ces auteurs qui ne répugnent pas à mouiller leur chemise en compagnie des déshérités du stylo» ${ }^{5}$. Dans tous les cas, l'écrivain est donc présenté comme "porte-parole», des obscurs, des muets, des marginaux, ...quand ce n'est pas d'une classe ouvrière qui aurait disparu.

Or, de telles lectures, convergentes et encore fréquentes aujourd'hui, ne sont pas totalement dénuées de pertinence. Tout d'abord, un simple regard sur les titres des romans semble conforter leur analyse: Sortie d'Usine, Décor Ciment, Temps Machine, Un fait divers, Parking, Mécanique... Les titres dessinent déjà un projet: rendre compte du monde moderne, de la société urbaine et industrielle. Et les personnages de ses romans, au premier abord, sont des victimes de ce monde qui les exclut: ouvriers, chômeurs, marginaux (C'était toute une vie, Prison, 30 rue de la Poste). D'autre part, François Bon lui-même anime des ateliers d'écriture, souvent destinés à des publics défavorisés, élèves des cités de banlieue, détenus, chômeurs en réinsertion: cette expérience a nourri certains de ses romans, et l'a amené également à en formuler la théorie et la pédagogie (Tous les mots sont adultes, Fayard 2000). Mais focaliser l'interprétation des textes sur leur contenu «social», (l'exclusion, la banlieue, le chômage, la désindustrialisation, etc.), sans être erroné à proprement parler, risque d'orienter la lecture vers une sorte de témoignage journalistique, au détriment du travail de l'écriture. Comme le dit très justement Yvan Leclerc, «il ne s'agit pas banalement de prêter sa voix aux hommes sans voix, mais de confronter l'exercice de la parole à sa limite mortelle: "ce qui en chacun gît inexprimé, et brûle d'être nommé « (Décor Ciment) " ${ }^{6}$. La difficulté, pour parler de l'œuvre de François Bon, est donc de croiser les deux exigences: l'indéniable recherche formelle, et la recherche du dehors, ce que l'auteur a appelé la «diction du monde» .

Le parcours biographique de François Bon n'est déjà pas typique, et ne ressemble pas en tout cas à celui de la plupart des écrivains de l'hexagone. De famille modeste mais cultivée (père garagiste, mère institutrice), il entame des études d'ingénieur aux Arts et Métiers, école prestigieuse qu'il quittera sans avoir le diplôme. (Il le relate dans Temps Machine, nous y reviendrons). Dès lors, il travaille en intérim, en région parisienne mais aussi à Moscou, à Bombay, à Prague, à Göteborg... Après avoir frôlé un accident grave, il renonce au travail industriel et se met à écrire. Quand paraît son premier roman, Sortie d'usine ${ }^{7}$, il n'a pas trente ans. A partir de ce moment, il se consacre à l'écriture, à la fois à son ceuvre personnelle et à l'animation d'ateliers.

${ }^{4}$ F. B on, Sang gris (1990), recueil de textes produits lors d'ateliers d'écriturc à La Courneuve (région parisienne): ce recueil ne figure pas dans la liste des ceuvres de l'auteur.

5 A. André, Bulletin de l'association Alcph, juin 1992.

${ }^{6}$ Y. Leclerc, Voir le vrai, tomber juste, "Critique» juin $1989, \mathrm{n}^{\circ} 503$

${ }^{7}$ F. Bon, Sortie d'usine, Editions de Minuit, 1982. 
Ce premier roman, paru aux Editions de Minuit, l'ambitieuse maison qui avait lancé le Nouveau Roman, relève-t-il de la même filiation? En un sens, c'est le cas, dans la mesure où l'on peut y voir les mêmes mises en question du personnage, de l'intrigue, de la gestion du temps... mises en question qui ont réuni sous la même étiquette, au prix encore de quelques malentendus, Robbe-Grillet, Butor, Sarraute et les autres. Comme dans les romans antérieurs rattachés à Minuit, si le titre Sortie d'usine annonce une histoire, ou au moins une diégèse, il est quelque peu trompeur. Polysémique d'abord, puisqu'il désigne aussi bien l'écriteau qui annonce un passage auquel il faut prendre garde (héritier en cela des surréalistes, qu'on se souvienne de Ralentir travaux) que l'acte accompli par l'auteur, de sortir de l'usine. Mais trompeur aussi parce que, d'histoire il n'y en a pas: seulement des segments de vie, fragmentés au long de quatre semaines, chacune sommant un chapitre, sauf le premier chapitre qui n'a pas de titre. Chaque semaine-chapitre est centré(e) sur un événement, mais ces événements se succèdent sans former une vraie continuité.

Le défi que se lance le texte consiste à raconter un laps de temps en usine, ni de façon militante, ni de façon misérabiliste, mais souvent sur le mode de l'énigme, pour dénaturaliser les évidences, et en général de façon behavioriste, ce qui là encore le rapprocherait du Nouveau Roman.

Dès le début du roman, s'impose au lecteur l'angoisse du narrateur anonyme, le stress du temps haché par le pointage: «sept heures un peu plus, c'est nuit encore» ${ }^{8}$, le minutage, l'anxiété devenue réflexe, «regard réflexe, dressé huilé», l'automatisation des gestes: «les arrêts qu'il ne comptait jamais et pourtant ne se trompait pas (...) L'arrêt jamais loupé, cette surprise d'avoir fait sans y penser les gestes...» (p. 12). Mais cette évocation n'est pas manichéenne, car le temps du travail, de la confrontation avec la matière, est aussi un temps positif, qui construit la personne en même temps qu'il façonne le matériau:

le travail même peut constituer la fuitc immédiate de l'ennui, ce qui s'achève et disparaît de l'établi laisse un vide qu'une pièce brute est déjà là pour emplir, et dont le brut même laisse voir, irréalisé mais présent, son fini, et sans commandement ni hâte oblige à la tâche (p. 37).

Chaque semaine-chapitre comporte, on l'a dit, un événement: la première semaine relate un accident, un ouvrier s'est blessé. L'évidence brute de la description (sang, pâleur du blessé, silence des machines) s'oppose au simulacre des rapports de force à l'intérieur de l'usine, l'obéissance obligée à la hiérarchie, la «revue du paraître» (p. 36), qui cesse dès que le narrateur sort de l'usine. «C'en est presque un plaisir, qu'obéir parfois soit ce théâtre et seulement cela, ces gestes sans effort qui ne sont que la caricature de ce qu'impose l'acien» (p. 36): la résistance de la matière, la réalité du travail, s'opposent cette fois au fantôme des rapports sociaux. Dans cette partie du récit apparaît un destinataire («voyez-vous»), un destinataire qui n'est pas au courant et qu'on informe ( Pas de suspension comme sur votre

\footnotetext{
${ }^{8}$ Ibidem, p. 7. Désormais les références de pages seront données dans le corps du texte.
} 
bagnole», p. 43) puis des énonciateurs pluriels, au nombre de quatre (p. 61-73), sorte de chœur antique qui préfigure l'importance, pour François Bon, de la tragédie.

Dans la deuxième semaine, un vieil ouvrier, revenu à l'usine pour une fête, meurt subitement. Ce n'est pas l'occasion d'un récit pathétique, mais d'une sorte de transfiguration: le mort inattendu, revêtu de son beau costume, est trimbalé sur un transpalette, chariot qui sert normalement au transport des matériaux. Cette scène, qui pourrait être burlesque, revêt une dignité quasi mythique, devient «le rite du Passage» accompagné par le vacarme volontaire que font les camarades du mort pour le saluer (p. 80-84) ${ }^{9}$, et le transpalette est explicitement assimilé à la barque de Charon traversant le Styx. On voit ici à quel point on est loin du «témoignage» brut sur la vie ouvrière, mais aussi à quel point l'auteur, qui a vécu cette vie, veut l'honorer en la parant des attributs que la mythologie réserve aux dieux.

Nous n'analyserons pas en détail la troisième semaine (la grève) ou la quatrième (où le narrateur expérimente le malaise, la maladie, la peur d'accidents, et décide de quitter). Indiquons seulement ce qui, dans ces derniers chapitres, définit le projet d'écriture qui se poursuivra dans les ouvrages ultérieurs. Lorsque le narrateur revient, en visiteur, à l'usine qu'il avait quittée, il prend conscience de ce qui devient visible parce qu'il n'y est plus: la prise de distance est indispensable à la création. Mais il est encore comme englué dans cette expérience, qui empêche l'émergence d'autre chose: «Ce qui pourrait être différent n'a pas encore commencé» (p. 167). Il se heurte aussi à la difficulté de figer, en la relatant, une réalité complexe et hétérogène: «Il y avait un vrai risque du constat à rassembler et figer ce qui était dispersé, ce qui ne se disait, ne s'affichait, que faute de savoir autrement l'exprimer» (p. 58). Et le roman s'achève de façon contrastée, sur une citation de vers d'Eluard, que la typographie transforme en prose: «Il y avait bien loin de ce château des pauvres Noir de crasse et de sang Aux révoltes prévues aux récoltes possibles» (p. 168), façon de réinscrire le texte dans une visée militante, fût-elle «assourdie», et d'autre part sur la phrase «Une sortie de tous les jours», qui éteint ou du moins tempère ce qui précède. Cette double polarité se retrouvera, avec des variantes, dans les romans suivants.

Il ne serait pas possible, dans le cadre d'un article, de consacrer une analyse aussi détaillée à tous les autres textes. On tentera seulement d'en repérer quelques récurrences, à la fois thématiques et esthétiques.

Si l'on s'en tient aux incipits, on constate que souvent, l'œuvre commence par l'annonce d'une mort. Description d'un cortège funèbre: «Les porteurs changent avec ceux qui tenaient les cordons, et ça s'ébranle à nouveau, tandis que le frisson du départ remonte lentement les dos. Les parents viennent devant, après eux un vide, et le cortège pris par le fouet ondulant des rafales s'effiloche jusqu'ici» ${ }^{10}$.

${ }^{9}$ A noter que ce vacarme rituel, pourtant hors contexte religicux, a son équivalent dans certaines survivances des rites catholiques en Méditerranée: le «vacarme des ténèbres» qui se fait durant les offices de la Semaine Sainte, en particulier le Vendredi Saint (commémoration de la mort du Christ).

${ }^{10}$ F. B on, L'Enterrement (Verdier 1992), p. 10. 


\section{Annonce d'un meurtre:}

Le mot planté. Le gardien-chef, alors que je sortais, ayant franchi la première porte-sas du bloc et repris ma carte d'identité, juste là devant le portique d'entrée à sonnerie, avant la porte verte à barreaux rectangulaires, près du portail pour le passage des fourgons cellulaires: «Et vous avez su que Brulin a été planté?»"

Visite à un cimetière:

Sa tombe à elle est ici, avec les dates: 1961 - 1993, et le prénom. Des choucas s'envolent en criant. Il n'y a jamais personne dans le cimetière (...) Sauf lui, qui vient le soir à dix-sept heures, avec ses deux chiens, a arrangé le ciment, le gravier blanc, la plaque et les fleurs ${ }^{12}$.

Ce qui relie ces quelques phrases, toutes placées à l'incipit du roman ou à proximité, c'est leur caractère énigmatique. Qui enterre-t-on? qui est Brulin, et pourquoi ce gardien-chef parle-t-il au narrateur d'un ton d'évidence, comme si celui-ci devait savoir? Qui est cette jeune femme, et qui est ce «Il» qui vient seul s'occuper de la tombe? Les énigmes seront apparemment levées dans les lignes qui suivent, on en saura un peu plus sur l'identité des disparus, sur leur relation au narrateur. Pourtant, une obscurité plus grande s'instaure peu à peu, qui n'est autre que le caractère fondamentalement inconnaissable des êtres. Si Alain de L'Enterrement s'est suicidé - et encore faudra-t-il, pour le savoir, traverser tous les mensonges de la famille, pour laquelle le suicide est un déshonneur à masquer --, le narrateur, pourtant ami proche, ne pourra nous dire pourquoi. Brulin a été poignardé «pour un motif futile» ${ }^{13}$. Quant à la jeune femme de C'était toute une vie, qui ne sera jamais nommée ${ }^{14}$, morte d'une overdose, elle a confié au narrateur une sorte de mission: elle lui a laissé quelques pages griffonnées, le priant de les rendre lisables ${ }^{15}$, et le narrateur se confronte à une difficulté qui ne tient qu'assez peu à la langue:

On est devant la tombe, on a les trois feuilles dans son sac, et on se dit qu'on ne saura pas. Que ce qu'il y a de savoir dans écrire ne tient pas à la maitrise de mots et comme on les arrange, mais à une autre experience, du corps et des yeux, du souffle, où c'est elle-même qui est devant. Qu'on ne saura pas, parce qu'on n'est pas allé où, elle, elle est allée. On se dit qu' on n'osera pas... ${ }^{16}$

${ }^{11}$ Idem, Prison (Verdier 1997), p. 7. Le mot planté signifie poignardé (argot),

${ }^{12}$ Idem, C'était toute une vie (Verdier 1995), p. 8.

${ }^{13}$ Titre du premier chapitse de Prison, et citation du fait-divers qui rendait compte du meurtre dans la presse locaie.

${ }^{14}$ Le hors-texte nous apprend qu'elle se prénommait Myriam, prénom que nous utiliserons désormais pour éviter les périphrases. Une adaptation théâtrale du roman, intitulée Vie de Myriam $M$., a été représentée à Paris en 1999.

${ }^{15}$ C'était toute une vie, op.cit, p. 8. «Lisable» est évidemment un barbarisme pour «lisible»: Myriam écrit un français fautif, question sur laquelle nous reviendrons.

${ }^{16}$ Ibidem, p. 10. 
Cette mission n'est pas propre à C'était toute une vie. En un sens, on peut affirmer que tous les personnages de François Bon lui en confient une: il s'agit à chaque fois de rendre compte de la complexité de leur monde. Tous ses romans sont des «biographies», biographies plus ou moins imaginaires d'êtres plus ou moins tirés de la réalité ${ }^{17}$. «Une vie n'est pas seulement la somme de témoignages contradictoires, mais un très grand silence, sur ce qui apparaît comme une solitude complexe: à chacun de nous elle présentait un visage, et la somme de ces visages ne suffit pas à la reconstruire» ${ }^{18}$.

Ce rapport à un deuil, et à la tentative de reconstruire une vie, la vérité d'un être, va toutefois prendre une autre ampleur avec un deuil plus personnel: la mort du père. Mécanique ${ }^{19}$, écrit après la mort du père, est à la fois livre de la perte, et tentative pour rassembler les fragments qui ont fait cette vie à présent terminée. Au même moment, François Bon a également publié Quatre avec le mort ${ }^{20}$, pièce de théâtre qui traite aussi d'un deuil familial, mais dont les protagonistes, avec leurs noms exotiques, Hirta, Dun, Boreray ${ }^{21}$, semblent bien éloignés de la réalité de la famille Bon. Cependant, François Bon a déclaré dans un entretien que «Quatre avec le mort va plus loin dans l'autobiographie, car le théâtre autorise à dépasser certaines censures: en se servant du corps des autres, on crée un écart par rapport à ce soi qui permet de l'écrire»" ${ }^{22}$.

Le texte s'écrit donc à partir d'une mort, comme C'était toute une vie, L'enterrement, Un fait divers, Décor Ciment ou Prison, mais il ne traite pas que de la mort d'un individu. Il s'agit aussi de faire le deuil d'un certain univers, «mécanique» au sens de la technologie, de mesurer le temps qui a passé aux traces désuètes des marques de voiture, des enseignes, de tout ce qui était les repères de l'enfant, fils et petit-fils de «mécanos». C'est par ces repères apparemment insignifiants, en tout cas objectifs, que se construit le souvenir: «surintensité des perceptions, de la remémoration, exacerbation de ce qui les relie l'un à l'autre» ${ }^{23}$.

Pour tracer ce portrait d'un père mort et d'un temps révolu, François Bon utjlise une structure qui n'a rien de chronologique, et dont la logique même n'apparaît pas au premier coup d'œil. Il s'agit d'une succession d'épisodes, annoncés par un mot ou un groupe de mots, isolés ou répétés, sans déterminations telles qu'articles, démonstratifs...: Voix, Lamento, Maison, Repères, Photo, Liste, Langue... etc. Sous la rubrique Photo, ce qui nous est décrit, ce ne sont pas des images de personnes ou

${ }^{17}$ Par exemple, Un fait divers est inspiré d'un fait divers réel, lu dans le journal, et la plupart des romans ont un substrat réel.

${ }^{18}$ C'était toute une vie, op.cit., p. 8.

${ }^{19} \mathrm{Idem}$, Mécanique (Verdier 2001).

${ }^{20}$ Idem, Quatre avec le mort (Verdier 2001). La pièce a cté crééc au Studio Théâtre de la Comédie Française en mars 2002.

${ }^{21}$ Il s'agit de noms d'ilôts des Hébrides, nous apprend une note liminaire.

${ }^{22}$ Propos recueillis par V. Marin La Meslée, Magazine littéraire, mai 2002, $\mathrm{n}^{\circ} 409$.

${ }^{23} \mathrm{~F}$. B on, Quatre avec le mort (Verdier 2001), quatrième de couverture. 
de paysages, mais d'engins motorisés: le père généralement n'y figure pas, c'est lui qui photographie, et cet effacement devant la machine le dépeint mieux que ne ferait un portrait. De même la rubrique Cartes postales: elles sont au nombre de quatre, envoyées pour quatre anniversaires du père par ses parents, et toutes représentent ... des voitures anciennes, avec des annotations biographiques de la grand-mère.

Mais ces rubriques peuvent donner l'impression d'une organisation préétablie, rigoureuse: or la mort du père est venue en quelque sorte bousculer un projet d'écriture, et lui donner corps, de façon imprévue. «Géométrie descriptive: on peut partir dans une écriture comme cela, sans notes ni préparation. J'y pensais depuis quelques mois, un livre qui aurait pu s'appeler «Maisons», juste comme ça des intérieurs, rien d'autre, pas de repères, pas de lieux, juste des figures de détail, ces meubles, des couloirs, escaliers, chambres.... ${ }^{24}$ La mort du père, que François Bon questionnait entre autres pour ce projet («à la fin du repas lui avais demandé ce que ça lui évoquait...»), fait ressurgir tout un passé resté brûlant: la «fuite» à Paris, après la démission des Arts et Métiers, la rupture avec les parents, qu'il ne voit pas pendant plusieurs années ${ }^{25}$, la déception d'une transmission interrompue, d'une trahison: le père allait pieusement aux 24 heures du Mans avec son propre père, les fils ne $s$ 'y sont guère intéressés («La légende était pour lui et pas pour nous») ${ }^{26}$, et l'auteur à son tour se rend compte qu' "(il) aurai( $t)$ du mal à attirer l'attention de (ses) propres enfants» sur ce légendaire mécanique si essentiel pour le père.

Le plus original de ce texte est peut-être le rapport à l'espace. L'espace est en effet décrit dans le temps, c'est-à-dire sous l'angle de ses transformations: les quelques éléments stables ne font que souligner l'entropie générale, «la force indifférente de ce qui est là sans que personne ne s'en préoccupe plus». Lorsque le narrateur et son frère arrivent dans le village où ils ont passé leur enfance, ils se perdent, ils ne reconnaissent plus rien. Les transformations objectives de l'espace sont aussi liées au temps personnel du narrateur, à sa propre évolution: «le bâtiment (...) semblait autrefois tellement plus grand ${ }^{27}$, «cette impression que si on écartait les bras on couvrirait plus large que tout ça, et même le mur, au fond, avec ses écroulements et ses brèches, on a l'impression qu'on le longerait en trois pas ${ }^{28}$. Il faut aussi remarquer qu'on va du microcosme au macrocosme: au début, le narrateur note les transformations dans le petit monde qui lui fut familier, puis c'est de plus en plus l'espace social, avec plus d'ampleur ${ }^{29}$. Enfin, comme dans les Artes Memoriae de l'Antiquité, c'est l'espace qui fait ressurgir le souvenir («cet étroit carré de ciment qu'on ne pouvait pas dire terrasse, mais c'est de lui que la mémoire

\footnotetext{
${ }^{24}$ Mécanique, op.cit., p. 47.

${ }^{25}$ Ibidem, p. 86.

${ }^{26}$ Ibidem, p. 118-120.

${ }^{27}$ Ibidem, p. 11.

${ }^{28}$ Ibidem, p. 21.

${ }^{29}$ Ibidem, p. 89 et passim.
} 
est faite ${ }^{30}$ ) et il est désigné comme ce qui fonde l'identité de la personne: «ce paysage de moteurs et de fer par quoi vous-mêmes avez assemblé votre perception extérieure du monde» ${ }^{31}$.

C'est de cet espace perturbé, perdu, que surgit peu à peu non pas un portrait du père, mais une silhouette en creux qui manifeste son absence. Sa mort n'est pas une surprise dans le texte (l'enterrement est présent dès le début) mais c'est une évidence subjective qui s'impose comme impossibilité à la mémoire: lui parti, c'est tout un pan de l'histoire familiale, et de l'histoire historique, qui devient à jamais inaccessible.

lui aurait pu nous le dire et ne nous le dira pas (...) on ne pourra sans lui être sû́ ${ }^{32}$,

l'idée que je ne savais pas pouvoir être fausse qu'on en reparlerait à volonte $e^{33}$.

Il n'est plus là pour me confirmer ${ }^{34}$.

Et c'est cela qui serait (...) votre dernière conversation? $?^{35}$.

Le fils en vient même à regretter l'époque des disputes: «il ne vous contredira plus jamais» ${ }^{36}$. Il se reproche de ne pas avoir su écouter l'angoisse de son père quand celui-ci parlait de sa mort prochaine:

et nous se moquant, lui disant qu'avec ses histoires il nous barbait, lui insistant que ce n'était plus comme avant, et nous rétorquant une fois de plus qu'il aimait trop à se faire plaindre et qu'à tout on pouvait résister, que c'était une question de décision intérieure, tenir et pas s'abandonner ${ }^{3}$.

En lisant ces lignes, quel lecteur peut se sentir innocent? n'avons-nous pas, chacun de nous, affirmé à quelque parent âgé qu' «il nous enterrerait tous», histoire de ne pas nous laisser contaminer par son angoisse?

Le père pourtant a écrit: il a laissé «quatorze pages» un peu comme Myriam dans C'était toute une vie, quatorze pages écrites avec trois encres différentes, mais ce n'est pas sa vie à lui, c'est le récit de la vie de son propre père. «Notes sibyllines parce qu'elles ne concernent que luì ${ }^{38}$ : contrairement à son fils, le père n'a pas l'idée de la communication, il n'a écrit que pour lui-même, dans un geste de piété intime envers son père à lui - piété que François Bon reconduit à sa manière, avec cette «stèle» qu'est Mécanique. Stèle au demeurant ambiguë ou ambivalente: le rêve de l'escalier si étroit que le père ne peut l'emprunter ${ }^{39}$ ne suggère-t-il pas

\footnotetext{
${ }^{30}$ Ibidem, p. 16.

${ }^{31}$ Ibidem, p. 14.

${ }^{32}$ Ibidem, p. 13-14.

${ }^{33}$ Ibidem, p. 31-32.

${ }^{34}$ Ibidem, p. 78.

${ }^{35}$ Ibidem, p. 33.

${ }^{36}$ Ibidem, p. 50.

${ }^{37}$ Ibidem, p. 20.

${ }^{38}$ Ibidem, p. 62.

${ }^{39}$ Tbidem, p. 92.
} 
l'impossibilité - pour chaque génération - d'accepter ce qui la précède et la suit tout en s'en différenciant?

Si la disparition d'un être est, on l'a vu, l'ébranlement initial de plusieurs des romans de Bon, cette disparition, dans le cas du père notamment, est étroitement corrélée à la disparition d'un univers. Poussant à l'extrême cette logique, l'auteur en vient à élaborer des textes dans lesquels le personnage humain a quasiment disparu. C'est le cas en particulier de Temps machine ${ }^{40}$ et surtout de Paysage $\mathrm{Fer}^{41}$.

Un des éléments les plus dépaysants pour nous de Temps machine, c'est que l'homme en est absent. «Le travail qui se fait seul», «les hommes en sont absents», «le peu qu'est un homme là-dedans» ${ }^{42}$ : de même que l'usine moderne tend à se passer de l'homme, le texte se passe du personnage, ou à peu près le premier mot du texte est «Fantôme»: double de l'homme disparu, revenant du passé, ou imaginaire, description de la machine qui l'élimine, et «des hommes le grouillement des métiers», foule anonyme. Or, pourtant, Temps machine est un texte profondément personnel, nourri de l'expérience vécue, et articulé sur un collectif: «un monde emporté vivant dans l'abîme, et nous accrochés au rebord, qu'il avait requis et modelé pour lui» ${ }^{43}$.

Ce qui meurt, c'est une époque, celle de l'industrialisation triomphante, c'est aussi une certaine image de l'histoire: le $\mathrm{XX}^{\mathfrak{e}}$ siècle a sonné le glas d'une civilisation qui s'était crue non seulement immortelle, mais potentiellement universelle. Les morts de la guerre et les morts de la guerre sociale sont explicitement comparés: «les noms (des morts) alors rajoutés au tableau à la porte de l'usine comme on avait fait après la boucherie sur les pyramides de ciment élevées sous l'emblème du coq ou du poilu, les hommes saisis dans le happement des presses ou le claquement des cisailles.... ${ }^{44}$.

Ce qui meurt avec le monde industriel, c'est une culture féroce pour les humbles, mutilante, dangereuse ${ }^{45}$, abêtissante ${ }^{46}$, mais qui avait ses valeurs, qui comportait ses qualités humaines et ses satisfactions: «la joie ou fierté encore à l'accomplissement mécanique» ${ }^{47}$. Comme dans Sortie d'usine, François Bon exhausse sa description à la mythologie:

${ }^{40} \mathrm{~F}$. B on, Temps machine (Verdier 1993).

${ }^{41}$ Idem, Paysage Fer (Vordier 2000).

${ }^{42}$ Temps machine, op.cit., p. 69-70 et p. 29.

${ }^{43}$ Ibidem, p. 93.

${ }^{44}$ Ibidem, p. 101. Ce qui était déjà présent dans le premier roman, Sortie d'Usine: «Ou quand au matin on lisait sur le panneau syndical l'avis avec les morts de la veille, Longwy" (p. 142).

${ }^{45}$ Temps machine, op.cit., p. 94 et passim.

${ }^{46}$ «Les bêtises (...) le goût à vomir du Ricard (...) les graffiti obscènes... (...) ces magazines idiots», les rêves dérisoires: «prendre un café tabac c'était après tout pour n'importe quel d'entre eux si beau rêve» (p. 17). Vie abêtissante aussi pour ceux qui ont un peu de pouvoir sur les autres, comme les contremaîtres: «Mort Dunand (...) après une vie empaillée...» (p. 99).

${ }^{47}$ Ibidem, p. 97. 
Morts en marche ils étaient: l'aiguilleur de Longwy comme sorti d'un livre dans le décor fantastique des cubilots déversant dans des bennes sur rails le métal en fusion... ${ }^{48}$

Cette mort est incompréhensible pour ceux qui en sont les acteurs ou plutôt les victimes: «même pour nous qui savions tout ça dans nos doigts était inimaginable la fin et la relégation du monde de fer et de tôle... ET LE TEMPS QU'IL FAUT POUR S'ÉTONNER DE TOUT ÇA» ${ }^{49}$. «A la mort même nous étions aveugles quand tout déjà s'écroulait...» ${ }^{50}$.

Comme Walter Benjamin analysant l'exception imaginaire que chacun de nous croit pouvoir réclamer pour lui-même dans l'universelle destruction ${ }^{51}$, Temps machine suggère qu'on ne peut pas penser sa propre mort, ou la disparition de son univers. Sans aucun passéisme: «Il n'y a pas à souhaiter de retour» ${ }^{52}$, hymne au progrès technique, mais un certain scepticisme qui invite à ne pas confondre progrès technique et progrès social: «tout va bien de par le monde sans doute qu'il s'améliore».

Cette vision critique du monde post-industriel s'enracine dans un des rares passages directement autobiographiques du texte, le premier chapitre intitulé «Fantômes». Le début du livre est aussi le début dans la vie, le temps des apprentissages et des dressages: or le narrateur refuse le bizuthage à l'entrée aux Arts et Métiers, ce qui équivaut à refuser le dressage à l'obéissance, refuser de devenir un de «ceux qui sauraient, dans l'atelier même et capables de se saisir du volant des machines, tenir face aux hommes en bleu la parole de qui profitait des mains mutilées et des vies prises» ${ }^{53}$. Même si ce refus est dur à tenir, et le laisse ambivalent: "pas fier ce jour là quatre ans de perdu rien dans les mains» ${ }^{54}$.

Cette révolte dicte la suite du livre: ayant rejeté «la parole de qui profitait des mains mutilées et des vies prises», le narrateur prend le risque d'être du côté de ces «vies prises». C'est aussi pour cela que le texte se passe de personnages: l'aliénation des vies, leur déshumanisation, ne permet pas qu'émerge un personnage au sens traditionnel. Le récit avance néanmoins, mais surtout par les lieux. La description des lieux est structurée par une vision sociologique (politique) du monde, par exemple pour Bombay: «pour parvenir au centre on remontait à l'envers toutes les couches de la ville jusqu'aux plus terribles»" ${ }^{55}$.

Nombre des lieux décrits sont désertés par l'activité humaine: «La tache blanche des machines enlevées et ferraillées... d'un coup de meule à disque » ${ }^{56}$. Et le

${ }^{48}$ Ibidem, p. 95.

${ }^{49}$ Ibidem, p. 98.

${ }^{50}$ Ibidem, p. 100.

${ }^{5 t}$ W. Benjamin, Sens unique (Maurice Nadeau 1975), passim.

${ }^{52}$ Temps machine, op.cit., p. 21.

${ }^{53}$ Ibidem, p. 12-13.

${ }^{54}$ Ibidem, p. 13. On a vu dans Mécanique la déception (implicite) du père quand le fils démissionne des Arts et Mêtiers.

${ }^{55}$ Ibidem, p. 31.

${ }^{56}$ Ibidem, p. 97. 
texte s'achève sur une apocalypse, qu'il faudrait pouvoir citer en entier, dont on ne donnera que les dernières lignes:

Le monde est fragile, et s'alourdit, les morts sont dans les immeubles et attendent (...) Le monde de formica tombera et les morts emmèneront avec eux ceux qui se contentaient d'aller au cinéma, lire leurs magazines, auront passé en abandonnant la révolte aux mains nơires qui n'en avaient plus la force et vivez donc, en attendant ${ }^{57}$.

La présence d'un destinataire, fût-il implicitement taxé de frivolité ou d'impuissance («et vivez donc, en attendant»), suffisait à notre avis à marquer ce texte d'un projet politique, ou social. Beaucoup plus sombre est Paysage fer, d'où cette fois tout personnage a bien disparu.

Le livre est dédié au photographe et plasticien Jérôme Schlomoff, dont les photographies, sous forme de diaporama ${ }^{58}$ puis de livre ${ }^{59}$, ont accompagné l'écriture. Il s'ouvre par une épigraphe empruntée à Michel Foucault: «C'est à notre sol silencieux et naïvement immobile que nous rendons ses ruptures, son instabilité, ses failles, et c'est lui qui s'inquiète à nouveau sous nos pas». La contrainte du livre est liée à un trajet en train, fait chaque semaine à la même heure sur le même trajet: le cadre spatio-temporel est donc strict, mais il est en même temps aléatoire, car la description des villes traversées, dans le désordre, ne suit pas la géographie, procurant ainsi une altération implicite du temps de l'histoire. L'auteur se donne néanmoins une règle précise: «Ne pas relire, accumuler seulement ces notations d'instants (...) Ne pas se contraindre à l'ordre (...) ne pas revenir sur ce qui s'écrit, accepter que ce texte, qu'on va reprendre tout l'hiver de semaine en semaine, commence de façon arbitraire après l'arrêt d'Epernay et qu'on y revienne selon la seule persistance rétinienne». Ces instants d'écriture viennent faire respirer ce que l'énumération des lieux traversés aurait, sinon, de suffocant.

Le paysage évoqué est un peu celui de Temps machine, la dimension internationale en moins ${ }^{60}$, et avec quelques années de décalage. C'est une France obsolète, celle de la révolution industrielle avec ses usines en friche, celle de la reconstruction de l'après-guerre avec ses kilomètres de béton. Descriptions apparemment hyperréalistes et dénuées de subjectivité, en fait très imprégnées des connaissances techniques et lexicales de l'auteur. Et qui viennent combler un manque théorique: «On a cherché, ces cinq mois, dans combien de librairies (...) les livres qui comporteraient des images de cela, des images de l'histoire des villes, des images de l'histoire des usines (...) Il n'y a rien. Cela apparemment n'intéresse pas la mémoire collective»" ${ }^{61}$. L'auteur fait lui-même quelques photographies, comme

${ }^{57}$ Ibidem, p. 104.

${ }^{58}$ Projeté le 2 avril 1999 au Centre dramatique de Nancy, pour accompagner la lecture d'extraits du livre, en avant-première.

${ }^{59}$ Aux éditions de l'Amourier, 2000.

${ }^{60}$ Temps machine évoquait les voyages professionnels de François Bon à Bombay, Moscou, Göteborg...

${ }^{61}$ Paysage fer, op.cit., p. 80-81. 
aide-mémoire, et relate avec humour l'hésitation du commerçant: "comme s'il se demandait si vous alliez accepter de payer, peut-être parce que vous aurez été déçu de cela qui vous est rendu (...) puisque sur aucune des quinze photographies aucun personnage visible, rien que cela: paysage fer, mais c'est bien ce qu'on voulait» ${ }^{62}$.

Les choses «manifestent encore leur imbrication au travail de l'homme»" ${ }^{63}$, mais si le texte s'achève sur un futur, c'est un futur vide:

La nouvelle ligne de train, enfin plus rapide, bientôt passera droit, il n'y aura plus que deux gares et quelques parkings. On sera nous-mêmes dispensés de constater l'abandon. On ne regardera même plus, peut-être, aux vitres du train ${ }^{64}$.

Cette écriture de l'extrême contemporain s'enracine néanmoins dans une tradition à la fois esthétique et linguistique. Nous ne pouvons ici qu'indiquer la relation essentielle, et plusieurs fois affirmée, de l'auteur aux tragiques grecs, qu'il relit avant d'entreprendre un nouveau texte ${ }^{65}$ : bornons-nous à esquisser brièvement le rapport des textes à la langue, et en particulier à la langue «fautive». François Bon écrit une langue spécifique, à la fois savante et populaire, tissée d'archaïsmes, de provincialismes, d' emprunts à l'oralité. Il s'en est expliqué à diverses reprises, non sans humour:

Je n'ai jamais senti la langue dans un rapport uni. Au contraire, une polyphonie de registres, et puis que ça racle, que ça brasse. (Rabelais, Céline...) Toujours comme quelque chose qui hurle derrière le bon ton. Le populisme, c'est d'aller dans le sens du poil, de laisser ça à plat. Le boulot, pour moi, serait de traiter ça en pur matériau plastique, par l'effet de compression qu'on peut en tirer. (...) Mon éditeur dit que j'écris un «français tordu», mais quand je lui demande où et quoi, ce qu'il me montre le plus souvent, c'est des formes reprises de Montaigne, ou des chroniqueurs du Moyen-Age $(. . .)^{66}$.

Ailleurs, il se réclame de la langue de Vendée «figée» et d'Agrippa d'Aubig$n e^{67}$, d'où viendrait entre autres «une manière d'arrêter la phrase trop tôt». Mais aussi «dans cette langue des choses, l'abandon trop souvent des verbes»: dès Sortie d'usine, le lecteur peut remarquer l'usage de l'infinitif, pour éluder les verbes conjugués qui présupposent un sujet, un Je, l'abondance des phrases nominales, l'emploi de pronoms que ne vient jamais compléter un nom propre.

${ }^{62}$ Ibidem, p. 81.

${ }^{63}$ Ibidem, p. 88.

${ }^{64}$ Thidem, p. 89.

${ }^{65} \mathrm{Cf}$. en particulicr Parking (Minuit 1996), texte de commande pour un téléfilm, en marge duquel l'auteur s'explique: “Comment Parking et pourquoi», p. 29-66. Il faudrait également évoquer toute l'intertextualité, extrêmement variée, qui nourrit les textes. Laurence Lombardi a consacré un mémoire de maîtrise aux citations dans Décor ciment, où elle repère notamment la présence de Rimbaud, de Baudelaire, de Rilke, de la Bible, etc. (Laurence Lombardi, Décor Ciment, De l'autre côté du rideau, 128 p. dactylographiées, Université de Provence I, 2001).

${ }^{66}$ F. B on, Côté Cuisines, entretien avec Sonia Nowoselsky-Müller, L'Infini no 19, été 1987.

${ }^{67}$ Mécanique, op.cit., p. 19 et 46. 
La recherche syntaxique et lexicale est étroitement liée au projet de compréhension du monde: «la revanche qu'on voulait de mots et d'une langue qui ressemble à tout ça» ${ }^{68}$. Le livre collecte, puis transforme, «l'hallucinante et maigre littérature» des bulletins d'usine, des affiches, des notes de service, des graffiti, «sans me rendre compte de la tâche inconsciente qui se manifestait là et s'y préparait par seule accumulation» ${ }^{69}$. Il affirme sa certitude de pouvoir apporter quelque chose de neuf, de jamais dit: «Que dans ce monde sans repère et en bascule on avait écrit bien trop et sur bien des choses, mais certes pas pour confier à la mémoire ce qu'était la fabrication des alternateurs ${ }^{70}$. D'où la présence massive de tout un langage technique, qui peut parfois déconcerter le lecteur «littéraire», mais surtout la valeur de réflexivité de ce langage: donnons-en un seul exemple, «et ne parlant que dégraissage, validant contre l'empire industriel en déconfiture la capacité des mots à soulever dans ce qu'ils représentent le masque de qui les nomme» ${ }^{71}$.

Réflexivité qui revêt aussi la forme d'une prise à la lettre des images verbales du langage courant, ainsi: «D'où il faudrait tirer la syntaxe qui tienne ceci à bout de bras (...) L'effort de tout tenir ensemble et comme il laisse aussi visible, par la grâce et tension de syntaxe, ce que l'autre expression «à bout de bras» ramène d'une nécessaire implication du corps» ${ }^{72}$.

C'est aussi ce que propose François Bon dans ses ateliers d'écriture, et qu'il met en œuvre dans ceux de ses romans qui s'appuient sur cette expérience. Dans ces romans, quand il lui arrive de citer ou de rapporter les paroles (ou les écrits) de ceux qui ne «maîtrisent» pas la langue, François Bon use de deux stratégies différentes. Soit il les rapporte textuellement, littéralement, en respectant les fautes: «jai des choses tres importante a te communiquer et je voudrais que tu larrange afin que ce soit lisable») ${ }^{73}$. Dans ce cas la faute, qui agresse en quelque sorte le lecteur, fait étroitement partie du message: elle signifie le parler de l'exclusion. A l'opposé, et le plus souvent dans un second temps, il lui arrive de «toiletter» les écrits fautifs: le lecteur une fois averti, maintenir la faute reviendrait à dévaloriser le propos du locuteur. Les deux stratégies, quoique opposées, ne sont donc pas contradictoires: toutes deux tendent à respecter la parole rapportée.

De tout ce travail, Tous les mots sont adultes propose une sorte de premier bilan:

Les ateliers d'écriture ont été pour moi le lieu d'une découverte surprenante du monde luimême, parce qu'en le nommant nous découvrons l'exigence pour l'écriture de s'ouvrir à des

\footnotetext{
${ }^{68}$ Temps machine, op.cit., p. 94.

${ }^{69}$ Ibidem, p. 100.

${ }^{70}$ lbidem, p. 74.

"Ibidem, p. 14.

72 Ibidem, p. 79.

${ }^{73}$ C'était toute une vie, op.cit., p. 8. Les termes soulignés le sont par nous: dans le texte, rien ne les différencie des formes «acceptables».
} 
syntaxes et des formes neuves, que ce réel neuf exige, et qui nous le révèlent en retour. (...) Plus qu'explorer l'univers de formes d'écriture préexistantes, explorer celui d'origines narratives à partir desquelles ce monde au dehors va appeler cette écriture formellement neuve qui nous aidera à constituer nos propres pratiques... ${ }^{74}$.

Nous espérons, par ces indications trop sommaires, avoir donné au lecteur l'envie de découvrir par lui-même l'œuvre si riche de François Bon.

\footnotetext{
${ }^{74}$ F. B on, Tous les mots sont adultes, op.cit., p. 7. Le titre est emprunté à Maurice Blanchot.
} 